\title{
Intoxicação alumínica na DRC
}

\section{Aluminium intoxication in chronic kidney disease}

\section{Autores:}

Fellype Carvalho

Barreto

Sonia M. Holanda

Almeida Araújo
1 Prevenção da intoxicação alumínica na DRC

1.1 Quelantes de fósforo $(\mathrm{P})$ à base de alumínio (Al) não devem ser utilizados em pacientes com doença renal crônica (DRC) (Evidência).

1.2 A concentração de $\mathrm{Al}$ na água e no dialisato deve ser monitorada semestralmente, sendo que essa concentração deve estar abaixo de $5 \mu \mathrm{g} / \mathrm{L}$ (Evidência).

2 Diagnóstico da intoxicação alumínica na DRC

2.1 O teste à desferroxamina deve ser realizado conforme indicação médica nos pacientes com DRC estágio V D (Evidência).

2.2 Pacientes com suspeita clínica de doença óssea relacionada ao $\mathrm{Al} \mathrm{e}$ teste à desferroxamina negativo devem ser submetidos à biópsia óssea (Evidência).

2.2.1 A presença de pelo menos $20 \%$ da superfície óssea trabecular recoberta por Al é considerada diagnóstica da intoxicação óssea por esse metal (Evidência).

2.2.2 A coloração histológica para a detecção de $\mathrm{Al}$ na superfície óssea deve compreender a coloração por solocromo-azurina, seguida pela coloração de Perls para afastar a presença de depósitos de ferro (Evidência).

3 Tratamento da intoxicação alumínica na DRC
3.1 O tratamento com desferroxamina deve ser feito na dose de $5 \mathrm{mg} / \mathrm{kg}$ de peso, por via intravenosa, diluída em $100 \mathrm{~mL}$ de solução glicosada a $5 \%$ ou fisiológica a $0,9 \%$, administrada durante 30 minutos, uma vez por semana, após o término da primeira sessão semanal de hemodiálise (HD) (Opinião).

3.2 Nos pacientes em diálise peritoneal (DP), a desferroxamina pode ser administrada por via intravenosa ou intraperitoneal, na dose de $5 \mathrm{mg} / \mathrm{kg}$ de peso, uma vez por semana (Evidência).

3.2.1 A infusão intravenosa deve ser feita durante 30 a 60 minutos, fora do período de diálise (cavidade abdominal vazia). A diálise só deve ser reiniciada após um mínimo de 5 horas do término da administração da desferroxamina (Opinião).

3.2.2 Caso opte-se pela via intraperitoneal, a desferroxamina deve ser adicionada à bolsa de maior permanência. Geralmente, na bolsa noturna para pacientes em diálise peritoneal ambulatorial contínua (CAPD) e na diurna para pacientes em diálise peritoneal automatizada (DPA) (Opinião).

3.3 O tratamento com desferroxamina deve ser interrompido ao final de 6 meses, e um novo teste deve ser repetido 1 mês após a interrupção (Evidência).

3.4 Em pacientes cujo teste à desferroxamina apresentar $\mathrm{Al}$ superior 
a $200 \mu \mathrm{g} / \mathrm{L}$, a desferroxamina deve ser administrada 5 horas antes da diálise, que deve ser realizada com membranas de alta permeabilidade (Evidência).

3.5 A administração de ferro intravenoso deve ser evitada, ou a dose reduzida, durante o tratamento com desferroxamina para impedir maior disponibilidade de ferro, favorecendo o aparecimento de infecções oportunistas (Opinião).

3.6 O tratamento com desferroxamina deve ser suspenso na vigência de sinais de toxicidade pela medicação e/ou de infecção (Evidência).

\section{RACIONAL}

O alumínio $(\mathrm{Al})$ é um dos metais mais abundantes na natureza e possui uma série de efeitos tóxicos nos seres humanos. ${ }^{1-4}$ Por ser de excreção predominantemente renal, essa toxicidade assume maior importância nos pacientes com DRC. Embora seja mais frequente em pacientes com DRC em diálise, a intoxicação por $\mathrm{Al}$ já foi descrita na DRC pré-dialítica e em pacientes transplantados renais. ${ }^{5,6} \mathrm{O} \mathrm{Al}$ acumula-se em vários tecidos, incluindo osso, cérebro, glândulas paratireoides e outros órgãos. ${ }^{1,2}$ Portanto, as manifestações clínicas do seu acúmulo são variadas, dependendo do órgão afetado, assim como da magnitude da intoxicação. Os principais sinais e sintomas da intoxicação alumínica são a anemia hipocrômica e microcítica, a neurotoxicidade aguda (agitação, confusão mental, mioclonia e convulsão), a encefalopatia dialítica (distúrbios da marcha e fala, apraxia motora, alucinações auditivas e visuais) e a doença óssea relacionada ao $\mathrm{Al}$ (osteomalacia e doença óssea adinâmica [DOA]).

As formas de contaminação por esse metal ocorrem por via oral, sobretudo pelo uso de quelantes de $\mathrm{P}$ à base de $\mathrm{Al}$, e a parenteral, através da água utilizada no preparo do dialisato. Outras fontes de contaminação descritas incluem o uso de utensílios domésticos de Al, ${ }^{6}$ soluções parenterais e alimentos. ${ }^{7,8}$ Além disso, o uso de medicamentos contendo citrato pode facilitar a absorção intestinal de Al. ${ }^{9} \mathrm{O}$ advento de quelantes de $\mathrm{P}$ que não contêm $\mathrm{Al}$ possibilitou a eliminação quase completa da via oral como fonte de exposição.

O emprego de sistemas mais sofisticados para a purificação da água (osmose reversa) minimizou o risco de intoxicação pela via parenteral em pacientes em HD. Por outro lado, estudos têm demonstrado que a exposição a pequenas concentrações de $\mathrm{Al}$, de forma contínua, pode levar à intoxicação por esse metal. ${ }^{10}$ Em um estudo que analisou biópsias ósseas realizadas em diversas regiões do Brasil, de 1985 a 2001, Araújo e cols. demonstraram que, embora a prevalência de intoxicação alumínica venha diminuindo (1985-1990: 61,3\%; 1991-1996: 38,7\%; 1997-2001: $42,5 \%$ ), ela ainda permanece elevada. ${ }^{11}$ Entretanto, por ser um estudo retrospectivo, as fontes de contágio não puderam ser analisadas. Valores de concentração de $\mathrm{Al}$ no dialisato de até $10 \mu \mathrm{g} / \mathrm{L}$ eram considerados seguros para se evitar a contaminação pelo metal. Atualmente, essa concentração é considerada inadequada, e uma concentração de $\mathrm{Al}$ menor que $5 \mu \mathrm{g} / \mathrm{L}$ parece ser a ideal. ${ }^{12,13}$ Além disso, estudos recentes demonstraram que a dosagem anual isolada é de pouca utilidade. ${ }^{14}$ Recomenda-se, então, que a monitoração da concentração de $\mathrm{Al}$ na água e no dialisato seja feita pelo menos semestralmente. Uma via adicional de contaminação que deve ser considerada são os sais utilizados no preparo do dialisato. Estudos que analisem o grau de pureza e a concentração segura de $\mathrm{Al}$ nesse importante componente do tratamento dialítico são necessários.

A dosagem de $\mathrm{Al}$ sérico pode ser útil para revelar uma exposição aguda a esse metal. No entanto, seu significado real e eficácia são questionáveis por não refletir a carga tecidual com precisão. ${ }^{15}$ Sherrard e cols. demonstraram que somente $50,1 \%$ dos pacientes com Al plasmático maior ou igual a $40 \mu \mathrm{g} / \mathrm{L}$ tinham DOA, enquanto que $14,2 \%$ daqueles com nível abaixo desse valor apresentaram DOA. Nesse estudo, utilizando-se como ponto de corte o nível de $40 \mu \mathrm{g} / \mathrm{L}$, a sensibilidade e a especificidade do Al plasmático para o diagnóstico de DOA foi de $65,2 \%$ e $76,7 \%$ respectivamente. ${ }^{16}$ Além disso, a interferência do estoque de ferro do paciente no nível sérico do $\mathrm{Al}$ é reconhecida. ${ }^{17-19}$ Pacientes em diálise com sobrecarga de ferro (ferritina $>500 \mathrm{ng} / \mathrm{mL}$ ) podem apresentar níveis séricos baixos de $\mathrm{Al}$ mesmo na vigência de uma carga corporal elevada, resultando em um teste à desferroxamina falso-negativo. Por outro lado, pacientes com deficiência de ferro (ferritina < $100 \mathrm{ng} / \mathrm{mL}$ ) podem apresentar níveis séricos elevados de Al, mesmo na ausência de acúmulo tecidual desse metal. ${ }^{15,20}$ Vale ressaltar que a alta variabilidade da concentração desse metal nas estações de tratamento de água dificulta a identificação de uma exposição aguda ao metal no paciente em HD, invalidando uma vez mais a dosagem isolada de $\mathrm{Al}$ sérico na DRC. O teste à desferroxamina, interpretado de acordo com os níveis de PTH e estoque de ferro, apresenta boas sensibilidade e especificidade para o diagnóstico da 
intoxicação pelo Al. Um teste à desferroxamina (5 $\mathrm{mg} / \mathrm{kg}$ ) positivo combinado com $\mathrm{PTH}<150 \mathrm{pg} / \mathrm{mL}$, com estoque de ferro adequado (ferritina entre 100 e $500 \mathrm{ng} / \mathrm{mL}$ ), tem valor preditivo positivo de $80 \%$ para doença óssea relacionada ao $\mathrm{Al} ;{ }^{21}$ ao passo que um teste positivo combinado com PTH $<650 \mathrm{pg} / \mathrm{mL}$ apresenta sensibilidade de $91 \%$ e especificidade de $95 \%$ para o acúmulo de $\mathrm{Al}$ no tecido ósseo. ${ }^{21}$

O teste à desferroxamina deverá ser realizado conforme indicação médica, em pacientes com DRC estágio V D que apresentem pelo menos uma das seguintes situações clínicas: presença de sinais e sintomas de intoxicação alumínica, início de tratamento farmacológico para HPS, antes de PTx e naqueles pacientes com história de exposição recente ao $\mathrm{Al} .^{22} \mathrm{O}$ teste é feito através de duas coletas de sangue, após 4 horas de jejum, para determinação dos níveis séricos de $\mathrm{Al}$, sendo a primeira coleta realizada antes da $1^{a}$ sessão e a segunda coleta antes da $2^{\text {a }}$ sessão de hemodiálise (HD) da semana. Após o término da primeira sessão de $\mathrm{HD}$, infundir a desferroxamina na dose de $5 \mathrm{mg} / \mathrm{kg}$ de peso, diluída em $100 \mathrm{~mL}$ de solução glicosada a $5 \%$ ou fisiológica a $0,9 \%$, durante 30 minutos. O teste à desferroxamina é considerado positivo se a diferença entre a $2^{\mathrm{a}}$ e a $1^{\mathrm{a}}$ concentração sérica de $\mathrm{Al}$ for $>50 \mu \mathrm{g} / \mathrm{L} .{ }^{21}$ Para pacientes em DP, o teste à desferroxamina deve ser realizado também com duas coletas de sangue para dosagem de Al sérico, separadas por um período mínimo de 5 horas, durante o qual, a DP deve ser interrompida (cavidade abdominal vazia). Sabe-se que, nesse período de 5 horas, a concentração de $\mathrm{Al}$ sérico atinge seu pico máximo após a infusão de desferroxamina. ${ }^{23}$

É importante ressaltar que se deve ter atenção especial na coleta de sangue e no método utilizado para sua dosagem. Por ser muito abundante na natureza, existe um risco elevado de contaminação da amostra, gerando resultados falso-positivos. Para se evitar tal interferência, a coleta deve ser feita em tubo seco livre de metal, enquanto a dosagem deverá ser feita pela técnica de espectrofotometria de absorção atômica, com forno de grafite. Embora o teste à desferroxamina tenha reduzido a necessidade de se realizar biópsia óssea, esta continua sendo o único método para o diagnóstico de certeza da intoxicação óssea pelo $\mathrm{Al} .{ }^{24}$ Assim, em caso de suspeita clínica de intoxicação por $\mathrm{Al}$ associada a um teste negativo, a biópsia óssea deve ser sempre realizada. A presença de pelo menos $20 \%$ da superfície óssea trabecular recoberta por Al, detectada através da coloração por solocromo-azurina, é considerada diagnóstica. ${ }^{25,26}$
A desferroxamina é amplamente utilizada desde 1960 como o único agente efetivo e específico para o tratamento de pacientes com hemocromatose, qualquer que seja a sua etiologia. Apenas em 1980 é que Ackrill e cols. relataram o emprego bem sucedido deste agente quelante de ferro no tratamento da intoxicação alumínica. ${ }^{27} \mathrm{~A}$ desferroxamina é uma sideroamina natural obtida a partir da cultura da bactéria Streptomyces pilosus. ${ }^{28}$ Sua substância ativa, o mesilato ou metanossulfato de desferroxamina $\mathrm{B}$, apresenta enorme afinidade pelo ferro trivalente, com capacidade de removê-lo da ferritina e da hemossiderina, mas não da hemoglobina. Ao se ligar ao ferro, forma o complexo ferrioxamina, substância hidrofílica que pode ser eliminada pelos rins e pela diálise. $\mathrm{O} \mathrm{Al}$ no sangue é pouco dialisável por ser amplamente ligado a proteínas, principalmente à transferrina. Análises in vitro detectaram uma elevação da fração ultrafiltrável do $\mathrm{Al}$ sérico após a infusão da droga, resultante da mobilização do $\mathrm{Al}$ dos depósitos tissulares e sua deslocação da transferrina com consequente elevação do $\mathrm{Al}$ sérico e formação de aluminoxamina, composto hidrossolúvel ultrafiltrável, com peso molecular de 583 D. Esta propriedade faz da desferroxamina uma droga de excelente ação mobilizadora e quelante do $\mathrm{Al}$ depositado nos tecidos, passível de remoção através das membranas utilizadas na diálise. Entre os tipos de membrana que contribuem para maior remoção do Al, destacam-se as de polissulfona e poliacrilonitrilo. ${ }^{29} \mathrm{~A}$ membrana peritoneal também é capaz de remover adequadamente o $\mathrm{Al}^{30} \mathrm{~A}$ hemoperfusão ou hemofiltração, por se tratar de procedimento dispendioso, são atualmente reservadas àqueles casos de intoxicação grave com manifestação clínica de neurotoxicidade. ${ }^{31}$

A dose de desferroxamina utilizada para o tratamento da intoxicação alumínica foi reduzida ao longo dos anos devido aos seus efeitos colaterais. Desde a Conferência de Consenso no Diagnóstico e Tratamento do Acúmulo de Alumínio na Doença Renal Crônica, em Paris, em 1992, preconiza-se utilizar uma dose de $5 \mathrm{mg} / \mathrm{kg}$, administrada uma vez por semana, após desligada a $1^{\text {a }} \mathrm{HD}$ da semana, por um período variável de 3 meses a 1 ano. ${ }^{32}$ Alguns autores demonstraram, por meio de estudos clínicos e farmacocinéticos, que doses mais baixas de desferroxamina $(<5 \mathrm{mg} / \mathrm{kg}$ de peso) podem ser eficazes no tratamento de pacientes com intoxicação pelo $\mathrm{Al} .{ }^{33-35}$ Porém, tais observações ainda precisam de maior confirmação antes de o seu uso ser recomendado. Nos pacientes submetidos à DP, a administração de desferroxamina pode ser feita por via intravenosa ou intraperitoneal, 
na mesma dose e frequência preconizadas para os pacientes em HD. ${ }^{36} \mathrm{~A}$ infusão intravenosa deve ser feita lentamente, durante 30 a 60 minutos, fora do período de diálise (cavidade abdominal vazia). A diálise só deve ser reiniciada após um mínimo de 5 horas após o término da administração da medicação. Caso optese pela via IP, a medicação deve ser adicionada às bolsas de maior permanência, ou seja, em geral na noturna, nos pacientes em diálise peritoneal ambulatorial contínua (CAPD), e na diurna, nos pacientes em diálise peritoneal automatizada (DPA). De modo geral, a desferroxamina é bem tolerada, porém não destituída de efeitos colaterais. Vários estudos relataram neurotoxicidade aguda dose-relacionada, exacerbação da encefalopatia alumínica, reações anafiláticas e maior suscetibilidade a infecções oportunistas, principalmente mucormicose. ${ }^{37-39} \mathrm{~A}$ ferrioxamina constitui nutriente para os microrganismos que utilizam ferro no seu metabolismo. Observou-se, experimentalmente, que a presença de ferrioxamina aumenta a taxa de proliferação de Rhizopus e reduz a eficácia terapêutica da anfotericina B. ${ }^{40}$ Nos últimos 10 anos, após a recomendação do uso de desferroxamina, na dose de $5 \mathrm{mg} / \mathrm{kg}$, os relatos de efeitos colaterais tornaramse esporádicos. ${ }^{6}$ Durante o tratamento com desferroxamina, a exacerbação do hiperparatireoidismo secundário pode ser observada devido à retirada do $\mathrm{Al}$ dos vários tecidos do organismo, principalmente paratireoides e osso. ${ }^{41,42}$ A hemoglobina e o volume corpuscular médio aumentam, indicando melhora da anemia, e a ferritina diminui em decorrência da ação quelante da medicação sobre os depósitos de ferro. ${ }^{43}$ O controle do tratamento pode ser feito por meio do teste de desferroxamina ou da biópsia óssea. ${ }^{44-46}$

\section{RefERÊNCIAS}

1. Alfrey AC, Hegg A, Craswell P. Metabolism and toxicity of aluminum in renal failure. Am J Clin Nutr 1980; 33:1509-16.

2. Alfrey AC. Aluminum metabolism in uremia. Neurotoxicology 1980; 1:43-53.

3. Drueke T. Dialysis osteomalacia and aluminum intoxication. Nephron 1980; 26:207-10.

4. Campbell A. The potential role of aluminium in Alzheimer's disease. Nephrol Dial Transplant 2002; 17(suppl 2):17-20.

5. Lin JL, Yang YJ, Yang SS, Leu ML. Aluminum utensils contribute to aluminum accumulation in patients with renal disease. Am J Kidney Dis 1997; 30:653-8.

6. Nicholas JC, Dawes PT, Davies SJ, Freemont AJ. Persisting aluminium-related bone disease after cadaveric renal transplantation. Nephrol Dial Transplant 1999; 14:202-4.

7. Mousson C, Charhon SA, Ammar M, Accominotti M, Rifle G. Aluminium bone deposits in normal renal function patients after long-term treatment by plasma exchange. Int J Artif Organs 1989; 12:664-7.

8. Oliveira SMR, Bertagnolli D, Bohre D et al. Serum aluminium: Influence of water and food consumed by patients with chronic renal insuficiency on regular hemodialysis treatment. J Bras Nefrol 2005; 27(3):101-109.

9. Drueke TB. Intestinal absorption of aluminium in renal failure. Nephrol Dial Transplant. 2002; 17(suppl 2):13-6.

10. Cannata-Andia JB. Reconsidering the importance of longterm low-level aluminum exposure in renal failure patients. Semin Dial 2001; 14:5-7.

11. Araujo SM, Ambrosoni P, Lobao RR et al. The renal osteodystrophy pattern in Brazil and Uruguay: an overview. Kidney Int Suppl 2003; (85):S54-6.

12. Cannata JB, Douthat W, Acuña G, Fernandez Martin JL. Aluminum toxicity: the role of prevention. Live Chem Rep 1994;11:207-13.

13. Fernandez-Martin JL, Canteros A, Serrano M, GonzálezCarcedo A, Díaz-Corte C, Cannata Andía JB. Prevention of aluminium exposure through dialysis fluids. Analysis of changes in the last 8 years. Nephrol Dial Transplant 1998; 13(suppl 3):78-81.

14. Jaffe JA, Liftman C, Glickman JD. Frequency of elevated serum aluminum levels in adult dialysis patients. Am J Kidney Dis 2005; 46:316-9.

15. van Landeghem GF, D'Haese PC, Lamberts LV et al. Low serum aluminum values in dialysis patients with increased bone aluminum levels. Clin Nephrol 1998; 50:69-76.

16. Kausz AT, Antonsen JE, Hercz G et al. Screening plasma aluminum levels in relation to aluminum bone disease among asymptomatic dialysis patients. Am J Kidney Dis 1999; 34:688-93.

17. Cannata JB, Olaizola IR, Gomez-Alonso C, MenéndezFraga P, Alonso-Suarez M, Diaz-Lopez JB. Serum aluminum transport and aluminum uptake in chronic renal failure: role of iron and aluminum metabolism. Nephron 1993; 65:141-6.

18. Cannata JB, Fernandez-Soto I, Fernandez-Menendez MJ et al. Role of iron metabolism in absorption and cellular uptake of aluminum. Kidney Int 1991; 39:799-803.

19. Huang JY, Wu MS, Wu CH. The effect of an iron supplement on serum aluminum level and desferrioxamine mobilization test in hemodialysis patients. Ren Fail 2001; 23:789-95.

20. Huang JY, Huang CC, Lim PS, Wu MS, Leu ML. Effect of body iron stores on serum aluminum level in hemodialysis patients. Nephron 1992; 61:158-62.

21. D'Haese PC, Couttenye MM, Goodman WG et al. Use of the low-dose desferrioxamine test to diagnose and differentiate between patients with aluminium related bone disease, increased risk for aluminium toxicity, or aluminium overload. Nephrol Dial Transplant 1995; 10:1874-84.

22. National Kidney Foundation: K/DOQI Clinical Practice Guidelines for Bone Metabolism and Disease in Chronic Kidney Disease. Am J Kidney Dis 2003; 42(suppl 3):S1-S202.

23. Verpooten GA, D'Haese PC, Boelaert JR, Becaus I, Lamberts LV, De Broe ME. Pharmacokinetics of aluminoxamine and ferrioxamine and dose finding of desferrioxamine in haemodialysis patients. Nephrol Dial Transplant 1992; 7:931-8. 
24. Moe S, Druke T, Cunningham J et al. Definition, evaluation, and classification of renal osteodystrophy: A position statement from Kidney Disease: Improving Global Outcomes (KDIGO). Kidney Int 2006; 69:1945-53.

25. Fernandez-Martin JL, Menendez P, Acuna G, Canteros A, Gómez C, Cannata JB. Staining of bone aluminium: comparison between aluminon and solochrome azurine and their correlation with bone aluminium content. Nephrol Dial Transplant 1996; 11 (Suppl 3):80-5.

26. Andress DL, Maloney NA, Coburn JW, Endres DB, Sherrard DJ. Osteomalacia and aplastic bone disease in aluminumrelated osteodystrophy. J Clin Endocrinol Metab 1987; 65:11-6.

27. Ackrill P, Ralston AJ, Day JP, Hodge KC. Successful removal of aluminum from patient with dialysis encephalopathy. Lancet 1980; 2:692-3.

28. Ackrill P, Day PJ. The use of desferrioxamine in dialysis associated aluminium disease. Contrib Nephrol 1993; 102:125-34.

29. Molitoris BA, Alfrey AC, Alfrey PS, Miller NL. Rapid removal of DFO-chelated aluminum during hemodialysis using polysulfone dialyzers. Kidney Int 1988; 34:98-101.

30. Hercz G, Salusky IB, Norris KC, Fine RN, Coburn JW. Aluminum removal by peritoneal dialysis: intravenous vs.intraperitoneal deferoxamine. Kidney Int 1986; 30:944-8.

31. Fernandez-Martin JL, Douthat W, Barreto S, Canteros A, Acuña G, Cannata Andía JB. Aluminium removal with the double chamber technique: paired filtrationdialysis (PFD).Nephrol Dial Transplant 1998; 13(supl 3):82-7.

32. Diagnosis and treatment of aluminum overload in endstage renal failure patients. Nephrol Dial Transplant 1993; 8 (supl1):S1-4.

33. Barata JD, D'Haese PC, Pires C, Lamberts LV, Simões J, De Broe ME. Low-dose $(5 \mathrm{mg} / \mathrm{kg})$ desferrioxamine treatment in acutely aluminium-intoxicated haemodialysis patients using two drug administration schedules. Nephrol Dial Transplant 1996; 11:125-32.

34. Canteros A, Diaz-Corte C, Fernandez-Marin JL, Gago E, Fernández-Merayo C, Cannata J. Ultrafiltrable aluminium after very low doses of desferrioxamine. Nephrol Dial Transplant 1998; 13:1538-42.
35. Kan WC, Chien CC, Wu CC, Su SB, Hwang JC, Wang HY. Comparison of low-dose deferoxamine versus standard-dose deferoxamine for treatment of aluminium overload among haemodialysis patients. Nephrol Dial Transplant 2010; 25:1604-8.

36. D' Haese PC, De Broe ME. Aluminum, lanthanum, and strontium. In: Daugirdas JT et al., editors. Handbook of dialysis. 4. ed. Philadelphia: Lippincott, Williams \& Wilkins; 2007. p. 714-26.

37. Olivieri NF, Buncic R, Chew E et al. Visual and auditory neurotoxicity in patients receiving subcutaneous deferoxamine infusions. N Engl J Med 1986; 314:869-73.

38. Sherrard DJ, Walker JA, Boykin J. Precipitation of dialysis dementia by deferoxamine treatment of aluminum related bone disease. Am J Kidney Dis 1988; 12:126-30.

39. Boelaert JR, Fenves AZ, Coburn JW. Deferoxamine therapy and mucormycosis in dialysis patients: Report of an international registry. Am J Kidney Dis 1991; 18:660-7.

40. Van Cutsem JV, Boelaert JR. Effects of desferoxamine, feroxamine and iron on experimental mucormycosis (Zygomycosis). Kidney Int 1989; 36:1061-8.

41. Morrisey J, Rothstein M, Mayor G, Slatopolsky E. Suppression of parathyroid hormone secretion by aluminum. Kidney Int 1983; 23:699-704.

42. Sedman AB, Alfrey AC, Milles NL, Goodman WG. Tissue and cellular basis for impaired bone formation in aluminium related osteomalacia in the pig. J Clin Invest 1987; 79:86-92.

43. Ott SM, Andress DL, Nebeker HG et al. Changes in bone histology after treatment with desferrioxamine. Kidney Int 1986; 18:S108-13.

44. Malluche HH, Smith AJ, Abreo K, Faugere M-C. The use of deferrioxamine in the management of aluminium accumulation in bone in patients with renal failure. $\mathrm{N}$ Engl J Med 1984;311:140-4.

45. Felsenfeld AJ, Rodrigues M, Coleman M, Ross D, Llach F. Desferrioxamine therapy in hemodialysis patients with aluminum associated bone disease. Kidney Int 1989; 35:1371-8.

46. Jorgetti V, Ricco-Soeiro NM, Mendes $\mathrm{V}$ et al. Aluminium-related osteodystrophy and desferrioxamine treatment: role of phosphorus. Nephrol Dial Transplant 1994; 9:668-74. 